\section{Chemin de Fer électrique monophasé de la Vallóo Brembana}

I.a vallée du Brembo, ou vallée Brembana, encaissée entre deux ranifications des Alpes, est une des plus pittoresques ot des plus accidentées de la Haute llalje; difrerents gros villages y sont disseminés, parmi lesquels San pellogrino qui est, en été, l'une des villes d'eau les plus fréquentees de litalie.

La vieille ville italienne de Bergame, dont la population est de 45000 hajilants, so trouve a la jonction de la vallee du Brembn el de celie du Serio.

Le développenent de l'industrie de la région, et l'important mouvement de voyageurs qui s'y produit l'éte, avait depuis longtemps rendu necessaire la creation d'un chemin de rer; mais la réalisation de celui-ci s'était j'usqu'à présent tronvée arrétée par les difficultés d'exécution quelle presentait.

C'est ce chemin do fer, qui vient atetro mis en exploitation, et sur loquel a té rénlisé une des premières applications du systeme de traction electrique par courant alternatif monophase, que nous nous proposons de décrire dans le present aricle.

I e chemin de fer de la Vallé Brembana réunit Bergame 217 metres d'allitude au village de San Giovanni Bianco (100 metros daltilude); sa longueur est de 30 kilometres. Outre les gares terminus de Bergame oi de San Giovanni Bianco, il y a sept gares intermédiaires . celles de Ponteranica, de Sombreno, de villa d'Alme, de sedrina, de 7ogno, d Ambria et de San Pellegrino. Toutes ces gares ont des voies de garage, el sont équipees pour faire le service des marchandises. Il y a cn outre plusieurs autres arrèts en cours de route.

La voie est simple à ecartement normal de $1^{\text {min }}$; les rails pèsent 27 kilogs au metre courant. L.e rayou minimum des courbes est de 150 metres; la pente maxima est de 24 pour mille, sur une longueur d'environ un kilometre.

La nature accidentee du terrain traversé a nécessité de nombreux ouvl'dres dat. Il y a 17 tunnels, dont quelquesuns ont une longuour dépassant 200 mètres; la ligne franchil le Bremho en plusieurs points.

l.es nombreuses chutes d'cau que présente la région ont fait envisager, des la mise en étude du chemin de fer, l'emploi de l'énergie électrique pout la traction des trains. Après examen des diférents systemes pouvant etre appliqués avec succes, la Societa per la Ferronia Lletrica di Valle Brembana se décida pour l'adoption du projet présenté par la sociste Westinghouse, basé sur l'emploi de la traction electrifue par courant monophasé à 25 périodes.

lsine géneratrice. - La station centrale hydro-electrique utilise une chute deau de 27 metres de hauteur, amenaréc sur le Brembo. Iille est située à 1000 métres en amont de San Giovanni Bianco, a San Pietro dorzio.

I.a station centrale produit du courant alternatif monophasé a 6000 volts, 25 périodes, au moyen d'alternateurs à inducteur tournant: ce courant est distribué aux voitures motrices au moyen d'une ligne de trolley a 6000 volts. Sur les locomolives, se trouvent des auto-transformateurs qui abaissent la tension de 6000 a 250 volts, tension d'alimentation des moleurs. Le retour du courant se fait par les rails, qui sont éclissés électriquement.

I.es groupes électrogenes pour la traction, au nombre de trois, se composent chacun dune turbine a axe horizontal, de la Maison Riva Monneret et $C$ de Milan, avec régulateur a circulation d'huile, tournant a 500 tours par minute, et dipectement accouplée, au moven d'un accouplement semi-elastique, a un alternateur Westinghouse de $500 \mathrm{k} w \mathrm{~s}$, a induit fixe et a inducteur tournant ( 6 pòles), produisant du courant a 6000 volts, 25 périodes, ainsi quil a été dejá dit.
L'excitation des alternateurs cst assurée au moyen de deux petits groupes electrogènes, tournant a 1000 tours par minute, et ayant chacun une puissance de $30 \mathrm{Kws}$ sous 110 volts.

Dans le but de rendre l'éclairage dans les gares absolument indépendant du service de la traction, un petit alternateur monophase a 25 périodes a cté prévu avec excitatrice en boul d'arbre. La puissance de ce groupe esl de $50 \mathrm{KVA}$. a 700 tours par minute. La périodicité et le vollage du service de l'celairage ont été choisis sumblables a la périodicité et an voltage du service de la traction, afin que le courant des groupes électrogenes de traction puisse éventuellement servir aussi pula l'eclairage.

Le tableau de distribution de la station centrale consiste en colonnes métalliques montées près de chaque machine. et portant les appareils el interruptcurs nécessaires à la commande de celte machine.

I a régulation du voltage des alternateurs de traction est. assuree au mogen d'un regulateur "Tirrill». dont le principe a cté décrit dans la Houille Blanche de juin 1907. On obtient ainsi une tension pratiquement constante, sur les barles de distribution, quelle que soit la charge et lo facleur de puissance.

I.es in ter: upleurs pour comrant alternatif sous 6000 volts sont a huile, et commandés a di-lance au moyen délectronimants parcourus par le courant continu de l'excitation. Le cablare de la station centrale est disposé dans lo soussol; il esl constitué par des barres de cuivre nu, montés sur isolateurs.

Le depart des lignes se fait du haut d'une tour situé sur lun des cotós du batiment; dans les difrerents étages de celte lour sont disposés los interrupteurs génèraux de la ligne, ainsi que les parafondres.

Lignes électriques. - A part la petite ligne qui relie la station centrale a la gare de San Giovanni Bianco, les lignes électriques comprennent quatro parlies distinctes:

10 I a ligne de contact proprement dite, avec fil de cuivre de $8 \mathrm{~mm}$. de diamètre; $2^{\circ}$ un feecier de méme section montésur les poteaux des linnes; 30 le circuit de retour, formé par les rails comectés électriquement; fo la ligne déclairage composée de deux fils de cuivre de $4 \mathrm{~mm}$. de diamétre.

Le fil de contact est suspendu, au moyen du systeme catenaire, à six metres au dessus du plan des rails. Le cable portour rst compose de sept brins dacier, ayant chacun un diamètre de 2 millimetres.

Les poleaux sont en bois de pin, injecté ou bichlorure do mercure. Normalement, la ligne est supportée par des barres transversales, supportees par deux poteaux: toutefois, dans certains cas speciaux, on a eté oblige liemployer. un seul poteau, avec des consoles qui ont la meme section et la meme forme que ces barres transversales: d'autre part, sur les ponts, on a remplacé les poteaux cn bois par une arche métallique. Dans les gares, à causc de la faible distance entre voics, on a été oblíré parfois de supprimer les poteaux intermediaires, et d'avoir des poutres transversales en treillis, dont la longueur atteint jusqu'a $18 \mathrm{~m}$.

I.e fil do trolley en cuivre est en forme de 8; il est suspendu au cable porteur au moyen de pendules de fer plat, et la griffe est en fonte malléable.

l.es portees normales de la ligne sont de 35 metres, avec fleche du cable portcur de $30 \mathrm{cms}$, ce qui correspond à une tension de 150 kilos à zéro denré; le nombre des pendules pour celte portée est de 14. In courbe, les portées sont reduites, de telle sorte que le désaxement de la ligne ne soit pas superieur a 50 cms au total, et des isolateurs d'ancrage sont prévus pour maintenir vertical le plan de suspension du fil.

En aliguement droit, on a également prévu de place en place des bras speciaux, pour eviter le lualancement du fil de contact autour du fil de suspension. 
Les points de fixage de la ligne de travail sont constilués par un systène de deux isolateurs speciaux : ces isolaleur. peuvent mésister dune tension continue de 15000 volts sous une pluie artificielle, et une tension instautanéc de 50 non volls. Ces deux isolateurs sont fixés à la partie supérieure d'une barre en fer, formee par doux fers a $U$ de $60 \mathrm{~mm}$. de hauteur, et pesant cnviron j $\mathrm{kg}$. 200 au metre courant. Dans les tunnels, les pinints de suspension de la ligne se trouvent situés tous les 15 mères dans la voute; la hauteur de la ligne au-dessus du plan des rails est de 4 m50.

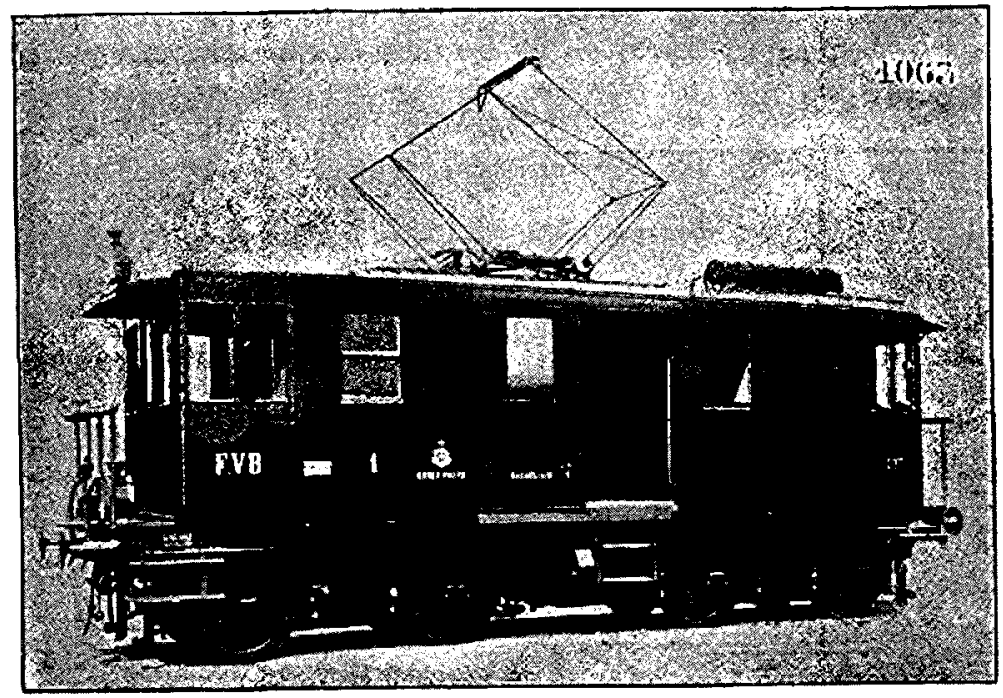

Fis. 1. - Vue duno locomotive.

Un feeder de $50 \mathrm{~mm}$. de section, connecté en paralllèle avec la ligne de traction, est spécialement prevu pour pouvoir facilement effectuer les llavaux sul une section, saus avoir a couper completement le courant. Dans toules les gares, sur des tours en treillis, se trouvent disposes des postes de sectionnement, qui permettent d'isoier, soit la ligne, soit le feeder, en cas de besoin.

Les interupteurs à huile sont commandés pardes renvois, ol peuvent ètre manceuvés par un ouvrier quelconque.

Dès que Yon isole une des deux sections de la ligne aboutissant à une gare, il est indispensable de couper tout courant sur la ligne do conlact de celte gare móme. Les locomotives électriques sont prévues, en effet, pour marcher accouplées; en double traction, la distance de leur trolley est denviron 11 mètres; si l'un des deux trolleys se trouvail dans le troncon precedant la gare, et rautre dans la gare mome, et si les locomotives ne prenaient pas de courant, la tension de hun des troncons se communicfunrait a lautre a travers los archets et appareils de contrólo multiple. In fil neutre, de 12 metres de long environ, évite la possibilité de mettre sous tension un ironçon de ligne sur lequel les ouvriers pourraient étre éventuellement a travailler.

Le retour du courant se fait par les rails; une seule ligne de rails est connecté électriquement au moyen de comexions électriques du genre Chicago, ayant une section de $50 \mathrm{~mm}^{2}$. Dans les aiguillages, ces connexions sont faites au moyen de tresses de cuivre d'une section beaucoup plus grande. La mise a la terre des rails a été assurée par des plaques de fer galvanisé, enfoncées d'environ 1 mo dans le sol. Ces plarjues sont disposées tous les kilometres.

La ligne de lumiere desserl des postes transformateurs établis daus les gares et dans les sections. Ces transformateurs sont disposés, soil sur une tour en treillis, soit sur deux poteaux jumellés.

Matériel roulant. - Un service de marchandises, ainsi qu'un service de yoyageurs, sont assurés yar 5 locomo- tives électriques a deux bugies moteurs. Ces locomalives, dont la partie mecanique a éte exécutée aux ateliers de la Societa cirnesto Bralit, sont equipees avec quatre moteurs électriques de 75 chevaux chacun.

Ces locomotives recuellent lénergie élcotrique au moyen d'un trolley panterraphe, qui permel de marcher dans ies deux sens sans manouvre spéciale. Le salrot de contacl, en aluminium. est appliqués contre le fil de la ligne au moyen de ressorts métaliiques disposes a la hase du trolley, dans un cylindre metallique, dit cylindre principal. En admettant l'air comprimé dans le cylindre. on agit contre les ressorts métaliques, et le trolley sabaisse. Lin dispositif de verrouillage a l'air comprimé permet de le fixer daus cette position, et d'isoler ainsi completement la voiture de la ligne.

Le transformateur monté sur la voiture est à un seul conoulement, ou auto-transformateur; une des extremiles de l'enroulement est connectée aux rails, lautre extrénité est connectes au trolley. Cet auto-transformatcur est ventilé artifciellement au moyen d'un petit ventilateur éect:ique alimonté par du courant monophase a 100 volls : une prise de courant est ménagée a cel effel sur lautotransformatour.

Le potentiel de l'enroulement du transformateur part du point zéro pour croitre at claque tour do l'enroulement de manière a ateindre de potentiel du trolley. Le systeme do controle consiste simplement a connecter les moteurs entre la terre of difrerents points choisis sur lenroulement des transformateurs. A cet effet, dess prises de courant, correspondant a des voltages propoltionnellement choisis, sont ménagés sur l'euroulement, et servent a graduer le voltage sur les bornes des moteurs. On obtient ainsi differentes variations de vitesse sans pertes rhéstaliques.

Pour passel de l'une des prises de courant a l'autre sans rompre le circuit, ou sans court circuiler les enroulements entre deux prises de courant considérés, il est nécessairo de prevoir un dispositir spe tal, tel quune résistanec ou

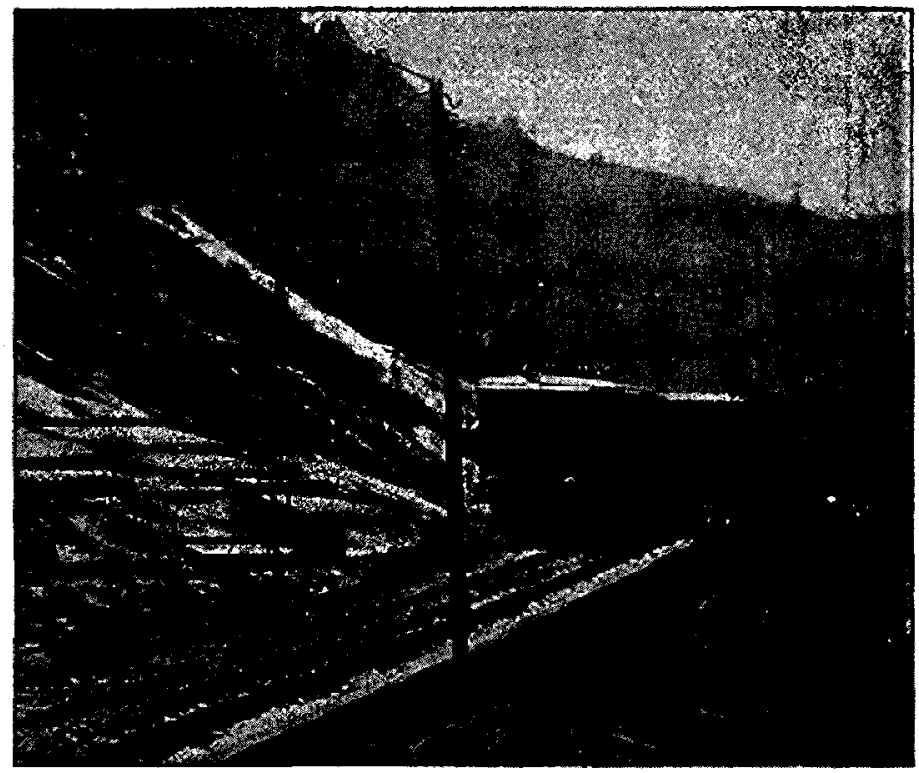

Fra. 2. - Vue de lis yoie el de la ligne acrienne.

bobine préventive, destinée à equilibrer le voltage des spires en court-circuit.

L.e contróle se fait au moyen de contacteur actionsés par l'air comprimé : l'air nécessaire à la fermeture des contacteurs est admis au moyen de valves électro-magnétiques. alimentées par courant alternatif monophasé á 50 volts. Ce dispositif evite l'emploi des batteries d'accumulateurs. Le wattman a en mains un petit controleur, dit controleur principal, qui commande le circuit des valves électro. máznétiques. 
Les moteurs électriques ont une puissance normale de 75 chevaux sous 250 volts, a la vitesse de 700 tours par minute; ils alaquent les essieux des roves par simple réduction d'engrenages, dans le rapport do 15 a 70 . Ces moteurs rappellent, par leur construction. les moteurs de traction employés avec le courant continu, el ils sont du type moteur série compense, a collecteur, et des dispositions palticulieres ont été imaginées pour assurer leur parfait fonctionnemeut.

Le nombre des circuits inducteurs est double : le premier est l'enrouloment série ordinaire, le second est un enroule. ment dit compensatour, gui engendre un fux perpendi culaire au flux produit pax le premier enroulement. Diutre
I a locomotive est équipéc avec lo frein Westinghouse; lair comprime necessaire à la manceuve de cot appareil est fourni par un petit compresseur dair mu par un moteur monophasé a 100 volts. Le réservoir principal d'air comprimé est placé sur le toit de la locomotive, les autres réscrvoirs ont été placés à l'intérieur.

l.es locomotives soul protegées contre les decharges atmospheriques par un parafoudre du type a Wurtz», place sur le toit. Entin, sur les enroulements du transformateur, une borne a 500 volts a été prévue pour le service d'eclairage et de chauffage des voitures a voyageurs.

Les locomotives ainsi equipees peuvent remorquer normalement un train de 90 tonmes, ontre Bergarne et San

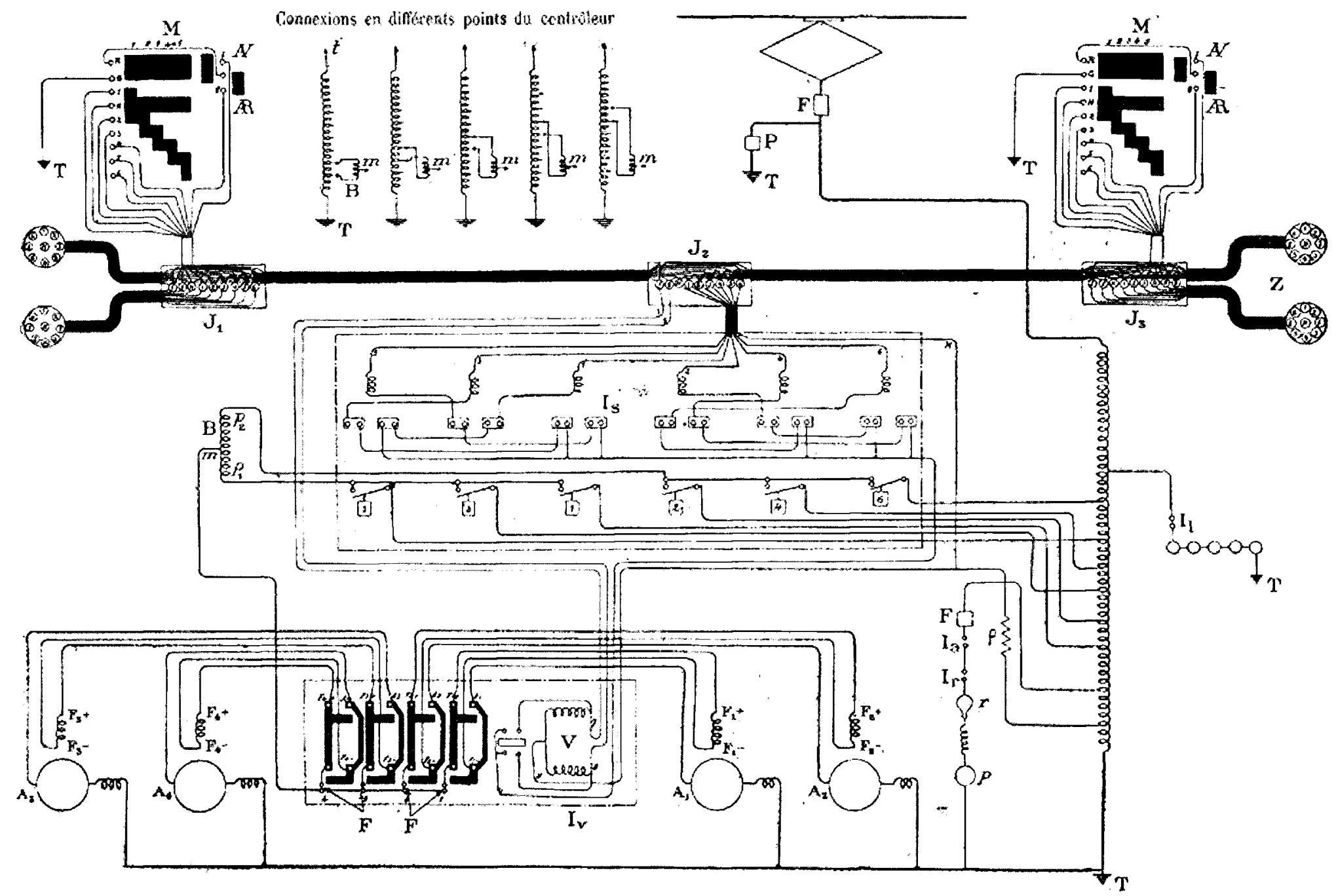

F16. 3. - Schoma des connections dune locomotive ilectrirue.

\section{工直GHIND四}

A. Moteurg detraction. 13. Bibine préveritivo.

13. Fusibles.

in Enroulement compengateur.

\author{
Is, Ir Interrupteurs des plate- \\ formes avant et arriere. \\ Il Interrupteur de lumière. \\ Is Interrupteurs secondaires tits \\ Ir inverseur.
}
J. Poites de jonction. M. Manipuiateur P. Pitaloudre.
$\checkmark$ Vilw maxnetigute
Z. Compleur lixe
T. "perre

m. Direction des moteurs.

p. Hompresseur.

p. Hisiktane onmigue.

$t$. Iirection did troiley. part, lorsqu'une spire est mise on court-circuit par un halai, te champ alternatif dans lequel elle se mout produit une force électro-motrice statique, qui cree un courant de courtcircuit intense. Pour limiter ces courants, et eviter les crachements aux balais, les connexions entre les bobines et los lamies du collecteur sont faites au moyen de conducteul's en maillechort très résistants. Ces conducteurs sont disposes au fond des cannelures; dun coté, ils sont solldés aux lames du collecteur, et du coté oppose au collecteur ils sont connectés aux bobines de linduit. Des anneaux degalisation rénissonc tous les points de l'induit au mème potentiel, et evitent les crachements aux balais lorsque vinduit vient à se decentrer.
Giovanni Bianco; ia vitesse maxima peut facilement atteindro 00 kilometres à theure.

Les essais qui ont été faits lors de la mise en service de ces locomotives ont permis de constater que, sur une rampe a 20 pour 1000 , on pouvait remorquer, a la vitesse de 18 kilomotres a l'heure, un train de 120 tonnes, of que. sur certaines parties du yarcours, le poids total du train remorqué pouvait atteindre 140 tonnes. Les essais dacceleration qui ont été poursuivis ont montré qu'il était possible doblenir des accélérations alloignant, et dépassant memo, 50 centimotros par seconde.

Le chemin de ter électrifue de la Vallée Brambana, par solt trafic et par lo tonnage de ses trains; ne saurait se 
comparer aux grandes lignes de chemin de fer; mais si lon tient compte que le systeme de traction monophasco permet facilement d'equiper les locomotives de 1000 chevaux et que d'autre part le voltasce au trolley de 6000 volts est loin d'ètre un maximum, et pourrait facilement etre porté a Vallee Brembana présente un grand intéret. Elle montre en petit ce que peut donner l'électricite, appliquée à la grande traclion, et particulièrement avec le systeme monophasé. A ce titre. cette installation mérite donc de retenir l'attenlion de tous les ingenieurs que seduit le probleme de lapplication de lélectricits a la traction des trains lourds.

\section{ButhION,}

Ingenieur Civit.

\section{Four contina à Induction}

M. Albert Hiorth, ingenieur a Cluristiania, a imaginé un noureau type de four a induction, permettant de marcher d'une manière coutinue, même pendan les réparations. Ce dispositit a fait l'objet du brevet français no 365.655 , du 23 avril 1906.

L'invention consiste en ce que l'apparcil électrique d'induction est agenci de manière a pouvoir servir aleernativemens, ou simulanement, pour deux fours. ou plus. Comme les fours nécessitent des réparations fréquentes et importantes, et qu'un

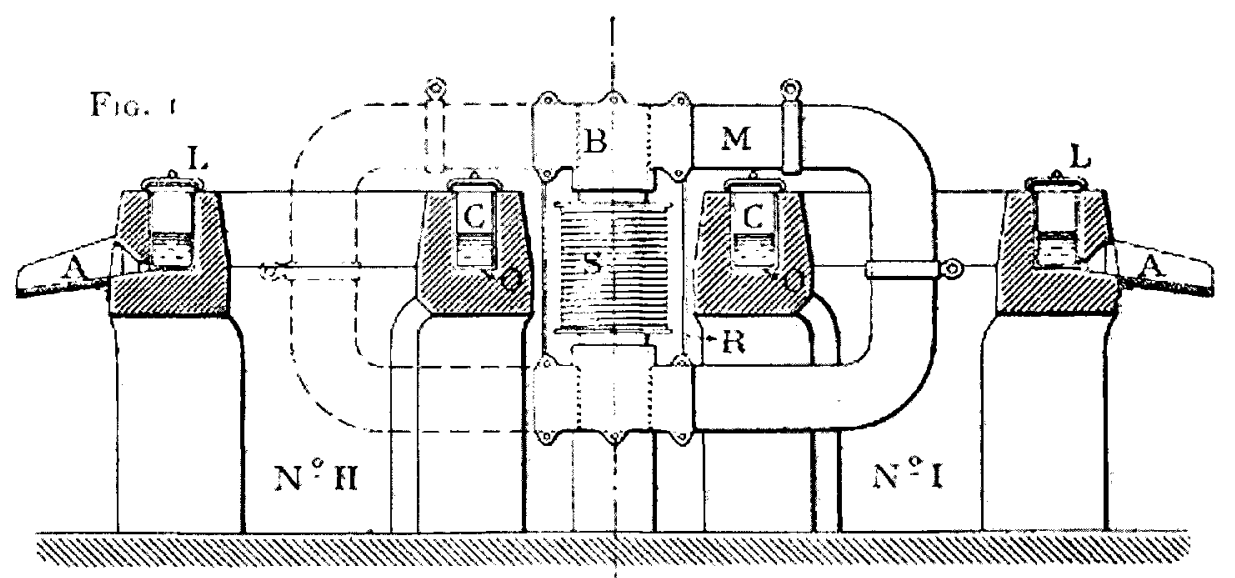
12000 volts, linstallation du chemin de fer élestrique de la

exemple, autour d'une bobine commune dinduction, on peut s'arranger, en cas de besoin, de façon te que trois des fours soient en marche pendant qu'on répare le quatrieme.

On peut aussi employer un étrier d'aimant commun pour deux fours, comme l'indique, à titre d'exemple, la fig. 2, auquel cas il est bon de disposer la bobine d'induction après l'étrier et

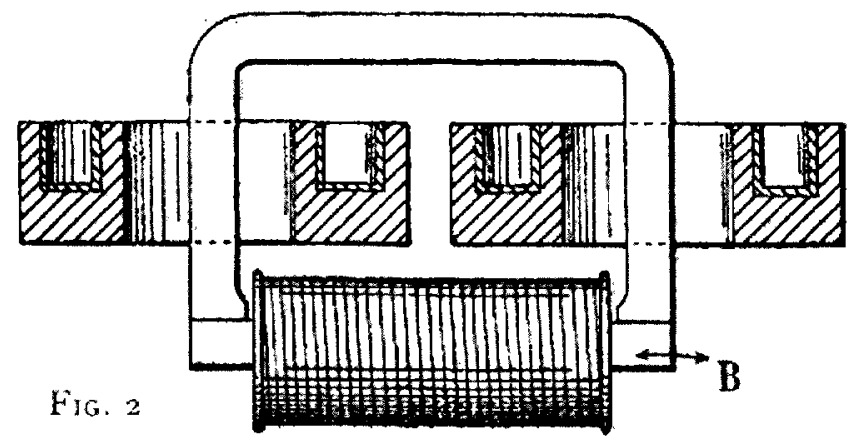

sous les fours. Dans cet agencement aussi, le noyau, avec sa hobine d'induction peut être détachable des pieces d'aimant $S$. de façon à pouvoir servir pour d'aurres fours.

La présente invention evite un autre inconvénient des fours d'induction employés jusqu'ici, savoir: l'obligation de vider entièrement les fours a chaque maconnement, et, pour remeture le four en marche, de mente, dans le four muni d'un revêtement neuf, une roue en métal coulé correspondant à l'espace interne du four, de manière a pouvoir etre fondue. Ceci n'est pas nécessuire dans les fours disposés conformément j la présente invention, car la charge est transféré directemen!, du foar qui doit être maçonne, dans les fours en étal de fonclionnement, et pell y être soumis au reste du traitcment, ce qui bconomise également de l'énergie.

In résumé, l'invention comprend : Le montage, dans les fours electriques defusion a induction, dine bobine commune d'induction pour deux fours, ou pius, dans lequel les partics partant de la bobitre dinduction som agences de manicre à pouvoir servir alternativement, ou simulanément, pour deux fours, ou plus, la ou les pièces d'aimant étant fixées au noyau de la nouveau masonnage doit secher lentement, l'appareil d'induction, dans les fours jusqu'ici connus, reste souvent longtemps inutilise, circonstance qui conduit a des frais d'exploitation elevés par rapport à la production. Pour éviter cel inconvénient, on monte, conformement a la présente invention, dcux fours, ou plus, $n^{\prime}$ I, $n^{\circ}$ II, à cóce, ou autour d'une bobine commune d'induction avec noyau $B$. Laimant $S$ qui, en sorlant du noyau $B$, passe par l'ouverure centrale du four $A$, est fixé au noyau B d'une façon détachable. Lorsque le four doit être réparé, on peut soulever l'aimant $S$ du four no I et le monter dans le four no 11 , en le fixant de l'autre côté du noyau $B$, comme on le voit par les lignes pointillées de la figure 1 .

On peut, at lieu de cet agencemen: du noyau B et de la piece d'aimant $S$, avoir une connexion déacbable entre ces partics, aux extrémités de la picce d'aimant $S$ montce dans l'ouverure centrale du four, el établir le noyau $B$ de façon à ce qu'il puisse tourner avec les branches qui en partent vers $S$. Dans ce cas, il peat $y$ aroir, en cas de besoin, une piece daimant $S$ fixe dans. chacun des fours.

Le métal est fondu, dans un creuset circulaice $C$, constitué par une garniture $O$ en briques réfractaires, sur lequel se traive un couvercle $L_{i}$, pour eviter les pertes de chaieur par rayopnement. La coulée se fait en $A$. Un grillage $R$ protège l'aimaat $S$ contre les radiations caloriftques dégagées par le four.

Si l'on a montó non pas deux, mais quatre fours, par bobine d'une maniere détachable (a cots ou autour de laquelle sont montés les fours) de façon à permetre d'enlever ou de fixer, suivant les besoins, la ou les pieces d'aimant, lorsquion. veut mettre l'un des fours hors d'activite.

\section{Les Services Météorologiques de la France}

EXTRAIT du rapport lu, le 22 mai 1907 , a la sénce gencrale du Conseil du burcau central molérologique, par. M. Bolquer de LA GrYs, memire de rinslilub, president.

Lorsque ce conseil du bureau central méborologique a be aviso: de la décision de M. Mascart de cesser ses fonolions do direcleur, son président, en son nom el en velui do tous ses collegues, a exprimé les regrets quo lour causail celle dibtermination inopine.

M. Bayet, au nom du minisire, formulait aussi tes memes regrets, at les faisait remonter bien au-dela de sa propre administralion, puisquill chail aulorisé à parler au nom de M. Liurd, son pridecesseur.

La nomillation de M. Mascart, comme directeur, remontat it 1818, a moment méme de la constitution de la métiorologie en un service autonome séparé de lohservatoire de paris. Physicien éminent, ses travaux sur l'oplique, l'électricité, le magnálisme. le designaient an choix du ministre, et il devait bientol se montrer administrateur habile, en dirigeanl successivement le premier établissement foudé rue de Grenelle, puis celui de la rue de l'liniversidé, qui peut étre considéré comme hors pair parmi les foudalions similaires en Europe. 